\title{
A Species Definition
}

\author{
RUTH E. GORDON \\ Waksman Institute of Microbiology, Rutgers, The State University of New Jersey, \\ Piscataway, New Jersey 08854
}

A definition of a microbial species, slowly evolved during years of work with many strains, is presented and explained.

Several months ago, a friend of long standing telephoned me and said in a very aggrieved tone, "I cannot find a definition of a species!" For the first time in his working life, this microbiologist needed a definition of a species, and he could not find one.

Microbiologists, especially taxonomists, talk about species, but a definition of a species, our unit of classification, is hard to find. The only definition that could be considered official can be found in the International Code of Nomenclature of Bacteria (5). The definition in this latest edition of the Code has not changed appreciably from that of the Proposed Code published in 1948 by Buchanan et al. (1). In its discussion of taxonomic categories-species, genus, family, etc.- the Code (Rule 5a) states: "... definitions of the taxonomic categories will inevitably vary with individual opinion...." Currently this is certainly a true statement of fact but, in my opinion, it is a worthless definition.

Since the species is our basic unit of classification, why is it that we have to leave the decision to the author? Is it because taxonomists are so independently minded, headstrong, opinionated, long-eared, and quarrelsome they cannot agree on such an important definition? Maybe so-speaking for myself!

However, I believe that there is a better explanation. In microbial taxonomy, our work, in Walter's (6) words, which I repeat at every opportunity, is providing the framework of communication among microbiologists by furnishing names of microorganisms and descriptions to give meaning to the names. We use the binary system of nomenclature, according to which a strain receives a species designation and a generic one. Related strains are assigned to a species and related species are assigned to a genus. Inherent in the binary syst $\mathrm{m}$ of nomenclature is the implication that microorganisms can be sorted into neat pigeonholes, into orderly groups with clear separations. (To digress a moment, I do not consider the binary system as sacrosanct. It can be replaced and it may be replaced in the future, but the replacement must be universally adopted and be one that will not disrupt communication among microbiologists, and I realize that will not be easily accomplished.)

Microorganisms do not lend themselves to arrangement in neat packages. As Cowan (3) wrote in Cowan \& Steel's Manual for the Identification of Medical Bacteria, "The different kinds of bacteria are not separated by sharp divisions but by slight and subtle differences in characters so that they seem to blend into each other and resemble a spectrum." The more strains and the more properties of the strains a taxonomist has studied, the more he is aware of the truth of Cowan's statement. This spectrumlike relationship of strains is, I believe, the cause of many of our difficulties, and it provokes endless controversy among taxonomists. If one examines only a few strains from each end of a spectrum of strains, it is easy to find differences between them. As a simple example, although the majority of strains of Nocardia asteroides in our collection (138 strains) do not grow at $45^{\circ} \mathrm{C}$ or form acid from rhamnose, there are 36 strains that are positive for these characters. In addition, there are 27 strains that are positive for one of the two characters and negative for the other. Observation of a few strains might support, and it has, the belief that strains of $N$. asteroides could be divided into two taxa, but examination of 100 strains would clearly demonstrate that growth at $45^{\circ} \mathrm{C}$ and acid formation from rhamnose are merely variable properties of $N$. asteroides.

In many laboratory notebooks, there are results of tests of a few strains of different species that gave promise of great usefulness in characterizing species, but, after application of these tests to more strains of each species, the great promise dwindled to nothing because we are dealing with spectra of strains or sections of a spectrum, not with compact packages of strains. In moments of greatest discouragement, I believe we taxonomists are attempting an impossible task.

Our work as taxonomists, on the other hand, fills a great need of all microbiologists: the need for names and reliable descriptions of the micro- 
organisms. It is thus high time we pulled up our socks and got on with it! Cowan (3) defined taxonomy as composed of three ingredients: (i) classification, (ii) nomenclature, and (iii) identification of the unknown. At present in taxonomy, the tail certainly wags the dog! Nomenclature, the least important ingredient and the least time-consuming, has its Code and Rules; classification, the most important ingredient of taxonomy, has nothing. In my opinion, one of the first things we need to do is to reach agreement, long overdue, on a definition of a species. The species is our unit of classification, and we greatly need a definition that takes into account the spectrum-like relationships of the strains to serve as a guiding principle for all of us in taxonomic work.

As a starting point for the reader's consideration, stones, or brickbats, I offer a definition of a microbial species first published in 1962 by Gordon and Mihm (4), one that I have repeated again and again. The wording has changed over the years, but the meaning is essentially the same. Currently the definition is as follows: A microbial species is a concept represented by a group of strains from a variety of sources, or by a population of strains, that contains freshly isolated strains, stock strains maintained in vitro for varying periods of time, and their variants (strains not identical with their parents in all characteristics [2]), which have in common a set, or pattern, of correlating stable properties that separates the group from other groups of strains.

What is the meaning of this definition? First, a species is represented by a population of strains or by many strains of different ages (considering their date of isolation as their birth dates) from a variety of sources. (One strain does not constitute a species; it is only one individual in a population, one point in a spectrum.) To delineate a species according to this definition, therefore, one must study many strains from a variety of sources and include newly isolated strains, old stock strains, and as many of their variants as possible; select the characteristics these strains have in common for describing the species they represent; and stress the similarities of all these strains, not their differences. If adopted, this definition would restrain investigators from creating new species without examining enough strains of possibly related species.

Anyone who works mainly with freshly isolated strains may be asking, "Why include old stock strains?" Old strains are important for a number of reasons. (i) Our nomenclature is tied to old strains or to strains that are rapidly getting older because the first or one of the first strains given a new species name by an author becomes the nomenclatural type strain of the species. Barring its demise, assignment of its name to the synonymy of another species, or, on rare occasions, action by the Judicial Commission of the International Committee on Systematic Bacteriology, the nomenclatural type strain must always belong to the species and be recognizable from the species description. In applying new tests and observations that result in the emendation of a species description, therefore, one must include the nomenclatural type strain. (ii) The old stock strains are also important in taxonomy because they represent the history of taxonomy or the work of taxonomists who preceded us, and history forms an important part of every branch of knowledge and experience. If some taxonomists knew more history, as exemplified by the strains available, I predict there would be fewer "new" species needlessly created. (iii) The most important reason, however, for including old strains in the taxonomic study of a species is the relative stability of their characteristics as compared with some of the characteristics of newly isolated strains. Microbial strains possess many properties of varying degrees of stability-one of the things that makes life interesting for us taxonomists. In my own experience, characteristics that persist after years of cultivation in test tubes are the more stable characteristics.

Why are variants (adapted strains or mutants) included in this proposed definition? Variants, developed in nature or in the laboratory, are part of a population of strains (like Mt. Everest, they are there), and they have as much right to a species name as the nomenclatural type or the newest isolate from nature. Variants, as well as old strains, can contribute to the determination of the more stable properties of the species. If, for example, one applies a test to a strain growing in the smooth stage and also to a rough variant of the same strain and finds that the smooth strain is positive and the rough variant is negative, the test is not likely to be a measure of a reliable property of the species. On the other hand, if one examines a goodly number of properties of a strain, obtains variants by laboratory manipulation from this strain, and applies to the variants the tests originally applied to the parent strain, the more persistent properties of the strain will be clearly evident. The selection of properties common to new isolates, old strains, and their variants will result in a more reliable description of the species than if only new isolations were observed and described.

Microbial variation and the spectrum-like relationship of the strains are always with us as two of the facts of life. We can compensate for 
these by including as many correlating properties of the strains as possible in the distinctive set, or pattern, of characters used to delineate the species. The distinguishing pattern of correlating properties must be large enough to permit variation by a strain in a few properties (one, two, or three perhaps) without excluding the strain from the species; that is, despite some variation, the overall pattern is that of the species. It is the similarities of the strains that are stressed rather than their differences.

Underlying our definition of a species is the firm belief that the species identity of a strain must always be inherent in the strain itself: not in its source of isolation, in the label on a test tube, in an investigator's notebook, or in the records of a culture collection, but in the properties of the strain. The description of a species should allow recognition of the species identity of a strain as long as the strain is extant. With the exception of the assignment of its name to the synonymy of another species name, as long as the strain is extant, it should bear the same species name.

So, feeling very much akin to the Man of La Mancha striving for the Impossible Dream, I am prepared to face reality and ask whether descriptions of species can be prepared as required by our proposed definition. For species widely represented in nature, it is not difficult. There are enough strains of well-represented species in culture collections and collections of investigators interested in the genus (often under a variety of names) to provide old stock strains; there are often replicates of the same strain in a number of collections to furnish spontaneous variants; and new isolations appear frequently. The more sparsely a species is represented in nature, the more difficult it is to find enough strains for the preparation of a species description according to our proposed definition. In some instances, a few more years are required to assemble enough strains; other species require a working life, whereas for others we can only present a description of a strain, or of a few strains, instead of describing a species. We can only attempt to do our best.

\section{REPRINT REQUESTS}

Address reprint requests to: Dr. Ruth E. Gordon, Waksman Institute of Microbiology, Rutgers University, P.O. Box 759, Piscataway, NJ 08854.

\section{LITERATURE CITED}

1. Buchanan, R. E., R. St. John-Brooks, and R. S. Breed. 1948. International bacteriological code of nomenclature. J. Bacteriol. 55:287-306.

2. Cowan, S. T. 1968. A dictionary of microbial taxonomic usage. Oliver and Boyd, Ltd., Edinburgh.

3. Cowan, S. T. 1974. Cowan \& Steel's manual for the identification of medical bacteria. Cambridge University Press, Cambridge.

4. Gordon, R. E., and J. M. Mihm. 1962. The type species of the genus Nocardia. J. Gen. Microbiol. 27:1-10.

5. S. P. Lapage, P. H. A. Sneath, E. F. Lessel, V. B. D. Skerman, H. P. R. Seeliger, and W. A. Clark (ed.). 1975. International code of nomenclature of bacteria. American Society for Microbiology, Washington, D.C.

6. Walters, S. M. 1964. Principles of taxonomy, p. 169-172. In C. F. Koda (ed.), Developments in industrial microbiology, vol. 5. American Institute of Biological Sciences, Washington, D.C. 